\title{
Analytic Solution for Nonlinear Multimode Beam Vibration Using a Modified Harmonic Balance Approach and Vieta's Substitution
}

\author{
Y.Y.Lee \\ Department of Architecture and Civil Engineering, City University of Hong Kong, Kowloon Tong, Kowloon, Hong Kong \\ Correspondence should be addressed to Y. Y. Lee; bcraylee@cityu.edu.hk
}

Received 29 June 2015; Accepted 7 October 2015

Academic Editor: Dumitru I. Caruntu

Copyright (C) 2016 Y. Y. Lee. This is an open access article distributed under the Creative Commons Attribution License, which permits unrestricted use, distribution, and reproduction in any medium, provided the original work is properly cited.

\begin{abstract}
This paper presents a modified harmonic balance solution method incorporated with Vieta's substitution technique for nonlinear multimode damped beam vibration. The aim of the modification in the solution procedures is to develop the analytic formulations, which are used to calculate the vibration amplitudes of a nonlinear multimode damped beam without the need of nonlinear equation solver for the nonlinear algebraic equations generated in the harmonic balance processes. The result obtained from the proposed method shows reasonable agreement with that from a previous numerical integration method. In general, the results can show the convergence and prove the accuracy of the proposed method.
\end{abstract}

\section{Introduction}

Over the past decades, many solution methods were developed for various engineering modelling problems (e.g., [14]). The two well-known solution methods, the perturbation method and multiple scale method (e.g., [5-7]), are widely adopted. In these two methods, coupled nonlinear algebraic equations are generated and required to be solved by nonlinear equation solver. There are two harmonic balance methods also widely employed (i.e., total harmonic balance method and incremental harmonic balance method, e.g., $[8,9])$. They also generate coupled nonlinear algebraic equations in the harmonic balance processes. In the previous works $[10,11]$, the multilevel residue harmonic balance method was modified from the total harmonic balance method and developed for nonlinear vibrations. Although the accuracies of these solutions were good and verified by other solution methods, it was quite time consuming to develop the nonlinear equation solver for those nonlinear algebraic equations generated in the solution procedures. On the other hand, there have been numerous beam/plate related research problems solved using various numerical and classical methods (e.g., [1215]). That is the motivation in this study to develop the analytic formulations to calculate the vibration responses of a nonlinear multimode beam without using a nonlinear equation solver for the nonlinear algebraic equations generated in the harmonic balance procedures. The proposed method is modified from the previous harmonic balance method and incorporated with Vieta's substitution technique. Using the analytic calculation formulations, the results can be obtained without the need of nonlinear equation solver.

\section{Governing Equation}

The governing equation of motion of nonlinear vibration of the Euler-Bernoulli theory is as follows [16]:

$$
E I W^{\prime \prime \prime \prime}+\rho_{A} \ddot{W}-\left(\frac{E A}{2 L} \int_{0}^{L}\left(W^{\prime}\right)^{2} d x\right) W^{\prime \prime}=F(x, t),
$$

where $x$ is longitudinal coordinate and $W$ is transverse displacement. $W^{\prime}, W^{\prime \prime}$, and $W^{\prime \prime \prime \prime}$ are 1st, 2nd, and 4th derivatives with respect to $x$, respectively; $\dot{W}$ and $\ddot{W}$ are 1st and 2 nd derivatives with respect to time, $t . L$ is length, $h$ is thickness, $E A$ is Young's modulus $\times$ cross-sectional area, $\rho_{A}$ is material density per unit length, and $F(x, t)$ is external harmonic excitation. For uniformly distributed excitation, $F(x, t)=F_{o} \sin (\omega t), F_{o}=\kappa \rho_{A} g=$ excitation magnitude, 
$\kappa$ is a dimensionless excitation parameter, $g=$ $9.81 \mathrm{~ms}^{-2}$, and $\omega$ is excitation frequency.

Then the governing equation is discretized using the modal reduction approach,

$$
W(x, t)=\sum_{i=1}^{n} q_{i}(t) \varphi_{i}(x)
$$

where $q_{i}$ is the modal amplitude of the $i$ th mode, $\varphi_{i}$ is the $i$ th structural mode shape, and $n$ is number of modes used.

Consider substituting (2) into (1) and multiplying $\varphi_{m}$ to it and taking integration over the beam length

$$
\begin{gathered}
\rho_{A} \sum_{i=1}^{n} \ddot{q}_{i} \alpha_{i, m}^{0,0}+E I \sum_{i=1}^{n} q_{i} \alpha_{i, m}^{4,0}-\frac{E A}{2 L} \sum_{i=1}^{n} \sum_{j=1}^{n} \sum_{k=1}^{n} q_{i} q_{j} q_{k} \alpha_{i, m}^{2,0} \alpha_{j, k}^{1,1} \\
-F_{m} \sin (\omega t)=0,
\end{gathered}
$$

where $\alpha_{i, m}^{0,0}=\int_{0}^{L} \varphi_{i} \varphi_{m} d x, \alpha_{i, m}^{4,0}=\int_{0}^{L} \varphi_{i}^{\prime \prime \prime \prime \prime} \varphi_{m} d x, \alpha_{i, m}^{2,0}=\int_{0}^{L} \varphi_{i}^{\prime \prime}$ $\varphi_{m} d x, \alpha_{j, k}^{1,1}=\int_{0}^{L} \varphi_{j}^{\prime} \varphi_{k}^{\prime} d x$, and $F_{m} \sin (\omega t)$ is modal force. For uniformly distributed excitation, $F_{m}=\int_{0}^{L} F_{o} \varphi_{m} d x / \int_{0}^{L} \varphi_{m}$ $\varphi_{m} d x$.

If the beam is simply supported, then $\varphi_{m}(x)=\sin ((m \pi /$ $L) x$ ) and $\alpha_{i, m}^{0,0}=\alpha_{i, m}^{4,0}=\alpha_{i, m}^{2,0}=\alpha_{i, m}^{1,1}=0$ for $i \neq m$. Then, (3) can be reduced into the following form:

$$
\rho_{A} \ddot{q}_{m}+\rho_{A} \omega_{m}^{2} q_{m}+\sum_{j=1}^{n} \beta_{m, j} q_{m} q_{j}^{2}-F_{m} \sin (\omega t)=0,
$$

where $\beta_{m, j}=-(E A / 2 L)\left(\alpha_{m m}^{2,0} / \alpha_{m, m}^{0,0}\right) \alpha_{j, j}^{1,1}$ and $\omega_{m}$ is the natural frequency of the $m$ th mode.

\section{Modified Solution Procedure Using Vieta's Substitution}

The solution form of the modal amplitudes is given by $[10,17]$

$$
q_{m}(t) \cong \varepsilon^{0} q_{m, 0}(t)+\varepsilon^{1} q_{m, 1}(t)+\varepsilon^{2} q_{m, 2}(t)+\cdots,
$$

where $\varepsilon$ is an embedding parameter; $q_{m, 0}(t), q_{m, 1}(t)$, and $q_{m, 2}(t)$ are the zero, 1st, and 2nd level modal amplitude solutions.

By inputting (5) into (4), consider those terms associated with $\varepsilon^{0}$ and set up the zero level governing equations

$$
\begin{aligned}
\rho_{A} \ddot{q}_{m, 0} & +\rho_{A} \omega_{m}^{2} q_{m, 0}+\beta_{m, m} q_{m, 0}^{3}-F_{m} \sin (\omega t) \\
+ & \sum_{j=1, m \neq j}^{n} \beta_{m, j} q_{m, 0} q_{j, 0}^{2}=\Delta_{m, 0}(t),
\end{aligned}
$$

where $q_{m, 0}=A_{m, 0,1} \sin (\omega t)$ and the first, second, and third subscripts in $A_{m, 0,1}$ are the mode number, zero level, and 1st harmonic number; $\Delta_{m, 0}$ is the zero level residual.
For simplicity, let

$$
\begin{aligned}
R_{m, 0}(t)= & \rho_{A} \ddot{q}_{m, 0}+\rho_{A} \omega_{m}^{2} q_{m, 0}+\beta_{m, m} q_{m, 0}^{3} \\
& -F_{m} \sin (\omega t) \\
S_{m, 0}(t)= & \sum_{j=1}^{n} \beta_{m, j} q_{m, 0} q_{j, 0}^{2} .
\end{aligned}
$$

Then, put (7a)-(7b) into (6)

$$
R_{m, 0}(t)+S_{m, 0}(t)=\Delta_{m, 0}(t) .
$$

Consider the harmonic balance of $\sin (\omega t)$ in $(7 a)$ :

$$
A_{m, 0,1}^{3}+\frac{4}{3 \beta_{m, m}} \rho_{A}\left(\omega_{m}^{2}-\omega^{2}\right) A_{m, 0,1}+\frac{4}{3 \beta_{m, m}} F_{m}=0 .
$$

When the damped vibration is considered, the zero level modal vibration amplitude is rewritten from (9) and its complex form is given by

$$
\begin{aligned}
& \left|A_{m, 0,1}\right| \\
& \quad=\left|\frac{F_{m}}{\prod_{m}(\omega)+(3 / 4) \beta_{m, m}\left|A_{m, 0,1}\right|^{2}+J\left(2 \xi \omega \bar{\omega}_{m}\right)}\right| .
\end{aligned}
$$

Then, (10a) can be rewritten as

$$
\begin{gathered}
\left(\frac{3}{4} \beta_{m, m}\right)^{2}\left|A_{m, 0,1}\right|^{6}+\frac{3}{2} \beta_{m, m} \prod_{m}(\omega)\left|A_{m, 0,1}\right|^{4} \\
+\left(\left(\prod_{m}(\omega)\right)^{2}+\left(2 \xi \omega \bar{\omega}_{m}\right)^{2}\right)\left|A_{m, 0,1}\right|^{2} \\
-\left(F_{m}\right)^{2}=0 .
\end{gathered}
$$

Consider a polynomial form

$$
a y^{3}+b y^{2}+c y+d=0,
$$

where, for simplicity, let $\prod_{m}(\omega)=\rho_{A}\left(-\omega^{2}+\omega_{m}^{2}\right)+$ $J\left(2 \xi \rho_{A} \omega \bar{\omega}_{m}\right), J=\sqrt{-1} ; \xi$ is damping ratio, and $\bar{\omega}_{m}$ is nonlinear peak frequency (note that, in case of linear vibration, $\bar{\omega}_{m}=$ $\left.\omega_{m}\right) ; y=\left|A_{m, 0,1}\right|^{2} ; a=\left((3 / 4) \beta_{m, m}\right)^{2} ; b=(3 / 2) \beta_{m, m} \prod_{m}(\omega)$; $c=\left(\prod_{m}(\omega)\right)^{2}+\left(2 \xi \omega \bar{\omega}_{m}\right)^{2} ; d=-\left(F_{m}\right)^{2}$.

Using the substitution of $y=\tau-b / 3 a,(10 c)$ can be further simplified as

$$
\tau^{3}+u \tau+v=0
$$

where $u=\left(3 a c-b^{2}\right) / 3 a^{2} ; v=\left(2 b^{3}-9 a b c+27 a^{2} d\right) / 27 a^{3}$; $\tau=B-u / 3 B$ (i.e., Vieta's substitution [18]). Equation (10c) can be rewritten as

$$
B^{6}+v B^{3}-\frac{u^{3}}{27}=0
$$

If $B^{3}$ is considered as an independent unknown, (12) is a "modified" quadratic equation. Hence, the analytic formula 
for the solutions can be obtained without the need of nonlinear equation solver. Then, the 1st level governing equation can be set up by inputting (5) into (4) and picking up those terms associated with $\varepsilon^{1}$ :

$$
\begin{aligned}
\rho_{A} \ddot{q}_{m, 1} & +2 \xi \rho_{A} \bar{\omega}_{m} \dot{q}_{m, 1}+\rho_{A} \omega_{m}^{2} q_{m, 1} \\
& +\sum_{j=1}^{n} \beta_{m, j}\left[\left(q_{j, 0}\right)^{2} q_{m, 1}+2 q_{m, 0} q_{j, 0} q_{j, 1}\right] \\
& +\Delta_{m, 0}(t)=\Delta_{m, 1}(t),
\end{aligned}
$$

where $q_{m, 1}(t)=A_{m, 1,1} \sin (\omega t)+A_{m, 1,3} \sin (3 \omega t)$.

Note that $A_{m, 0,1}$ has been found from the zero level solution procedure. Thus, $q_{j, 0}$ and $\Delta_{m, 0}(t)$ in (13), which are in terms of $A_{m, 0,1}$, are known. Therefore, (13) is a linear equation.

Consider the harmonic balances of $\sin (\omega t)$ and $\sin (3 \omega t)$ in (13) to set up two algebraic linear equations

$$
\begin{gathered}
\int_{0}^{2 \pi} \Delta_{m, 1}(t) \sin (\omega t) d t=0 \\
\int_{0}^{2 \pi} \Delta_{m, 1}(t) \sin (3 \omega t) d t=0
\end{gathered}
$$

As (14a)-(14b) are linear, the unknown modal amplitudes $A_{m, 1,1}$ and $A_{m, 1,3}$ can be found analytically.

Again, the 2 nd level governing equation can be set up by inputting (5) into (4) and picking up those terms associated with $\varepsilon^{2}$ :

$$
\begin{aligned}
& \rho_{A} \ddot{q}_{m, 2}+2 \xi \rho_{A} \bar{\omega}_{m} \dot{q}_{m, 2}+\rho_{A} \omega_{m}^{2} q_{m, 2} \\
& +\sum_{j=1}^{n} \beta_{m, j}\left[q_{m, 0}\left(q_{j, 1}\right)^{2}+2 q_{m, 0} q_{j, 0} q_{j, 2}+q_{m, 2}\left(q_{j, 0}\right)^{2}\right. \\
& \left.\quad+2 q_{m, 1} q_{j, 0} q_{j, 1}\right]+\Delta_{m, 1}(t)=\Delta_{m, 2}(t)
\end{aligned}
$$

where $q_{m, 2}(t)=A_{m, 2,1} \sin (\omega t)+A_{m, 2,3} \sin (3 \omega t)+$ $A_{m, 2,5} \sin (5 \omega t)$.

Note that $A_{m, 1,1}$ and $A_{m, 1,3}$ have been found from the 1st level solution procedure. Thus, $q_{j, 1}$ and $\Delta_{m, 1}(t)$ in (15), which are in terms of $A_{m, 1,1}$ and $A_{m, 1,3}$, are known. Therefore, (15) is a linear differential equation.

Consider the harmonic balances of $\sin (\omega t), \sin (3 \omega t)$, and $\sin (5 \omega t)$ in (15) to set up three algebraic linear equations:

$$
\begin{aligned}
& \int_{0}^{2 \pi} \Delta_{m, 2}(t) \sin (\omega t) d t=0 \\
& \int_{0}^{2 \pi} \Delta_{m, 2}(t) \sin (3 \omega t) d t=0 \\
& \int_{0}^{2 \pi} \Delta_{m, 2}(t) \sin (5 \omega t) d t=0
\end{aligned}
$$

As (16a)-(16c) are linear, the unknown modal amplitudes $A_{m, 2,1}, A_{m, 2,3}$, and $A_{m, 2,5}$ can be found analytically. Similarly, the $r$ th level governing equations can be set up by inputting
TABLE 1: (a) Convergence study for various excitation magnitudes, $\omega=1.5 \omega_{1}$. (b) Convergence study for various excitation frequencies, $\kappa=7.5$.

(a)

\begin{tabular}{lcccc}
\hline $\begin{array}{l}\text { Normalized overall } \\
\text { amplitude }\end{array}$ & $\kappa=1$ & 7.5 & 15 & 25 \\
\hline Zero level solution & 99.92 & 101.00 & 101.93 & 102.69 \\
1st level solution & 100.00 & 100.03 & 100.13 & 100.32 \\
2nd level solution & 100.00 & 100.00 & 100.00 & 100.00 \\
\hline
\end{tabular}

(b)

\begin{tabular}{lcccc}
\hline $\begin{array}{l}\text { Normalized overall } \\
\text { amplitude }\end{array}$ & $\omega=0.5 \omega_{1}$ & $\omega_{1}$ & $3 \omega_{1}$ & $6 \omega_{1}$ \\
\hline Zero level solution & 99.79 & 100.44 & 96.43 & 79.43 \\
1st level solution & 100.03 & 100.03 & 100.00 & 100.00 \\
2nd level solution & 100.00 & 100.00 & 100.00 & 100.00 \\
\hline
\end{tabular}

(5) into (4) and picking up those terms associated with $\varepsilon^{3}, \varepsilon^{4}, \varepsilon^{5}, \ldots$ and consider the higher level solution form

$$
\begin{aligned}
q_{m, r}(t)= & A_{m, r, 1} \sin (\omega t)+A_{m, r, 3} \sin (3 \omega t) \\
& +A_{m, r, 5} \sin (5 \omega t)+\cdots .
\end{aligned}
$$

Then, consider the harmonic balances of $\sin (\omega t)$, $\sin (3 \omega t), \sin (5 \omega t), \ldots$ to set up the linear algebraic equations and solve for the unknown modal amplitudes $A_{m, r, 1}$, $A_{m, r, 3}, A_{m, r, 5}, \ldots$

Finally, the overall amplitude and overall modal amplitude are defined as

$$
\begin{aligned}
A_{\text {over }} & =\sqrt{\sum_{m} \sum_{r}\left|\sum_{s} A_{m, r, s}\right|^{2}}, \\
A_{\text {over }, m} & =\sqrt{\sum_{r}\left|\sum_{s} A_{m, r, s}\right|^{2}},
\end{aligned}
$$

where $m$ is mode number (i.e., $1,2,3, \ldots$ ), $r$ is solution level (i.e., $0,1,2, \ldots$ ), and $s$ is harmonic number (i.e., $1,3,5, \ldots$ ).

\section{Results and Discussions}

In this section, the material properties of the simply supported beams in the numerical cases are considered as follows: Young's modulus $E=71 \times 10^{9} \mathrm{~N} / \mathrm{m}^{2}$, mass density $\rho=2700 \mathrm{~kg} / \mathrm{m}^{3}$, beam dimensions $=0.5 \mathrm{~m} \times 0.2 \mathrm{~m} \times$ $5 \mathrm{~mm}$, Poisson's ratio $=0.3$, and damping ratio $\xi=0.02$. Tables 1(a)-1(b) show the convergence studies of normalized beam vibration amplitude for various excitation magnitudes and frequencies. The two-mode approach is adopted. The harmonic excitation is uniformly distributed. The 2 nd level solutions are normalized as one hundred. It is shown that the 1st level solutions are accurate enough for the excitation frequency range from $0.5 \omega_{1}$ to $6 \omega_{1}$ and excitation parameter from $\kappa=1$ to 25 . The differences between the zero 


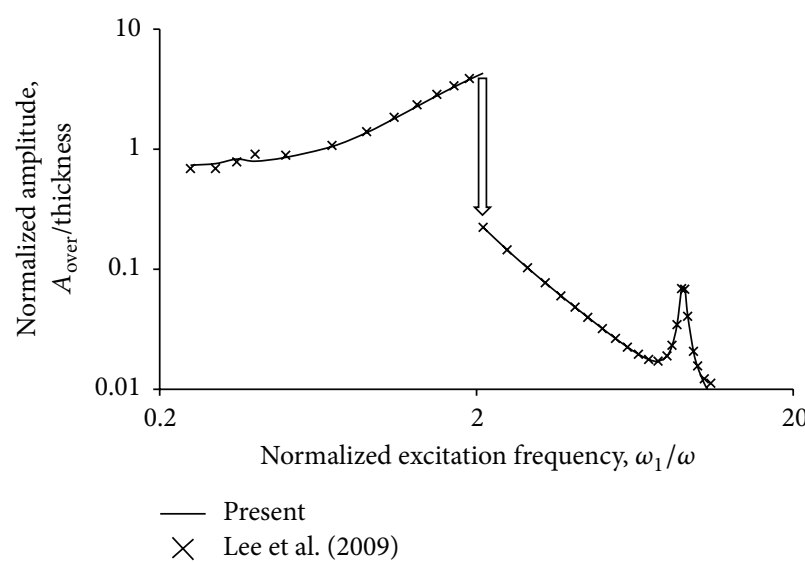

(a)

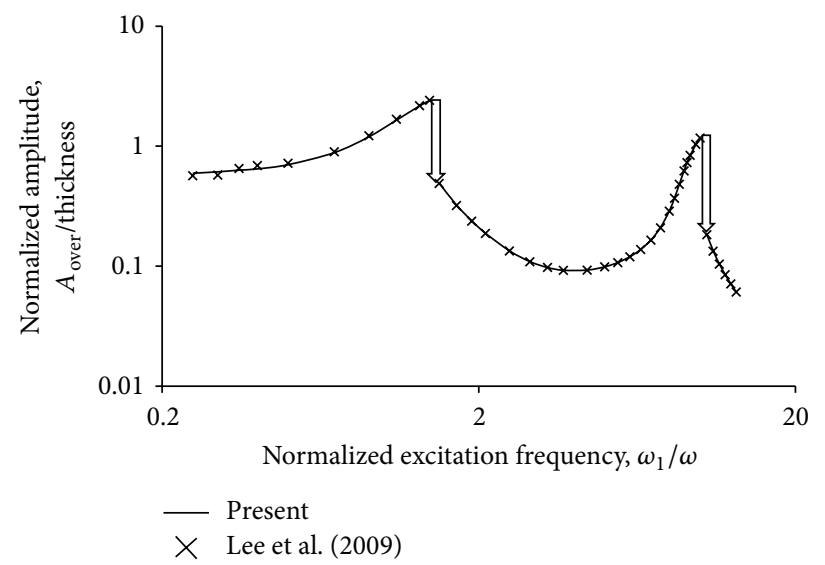

(b)

Figure 1: (a) Comparison between the results from the proposed and numerical integration methods, $F=7.5 \rho_{A} g \sin (\omega t)$. (b) Comparison between the results from the proposed and numerical integration methods, $F=75(0.1 \sin ((\pi / L) x)+0.9 \sin ((3 \pi / L) x)) \rho_{A} g \sin (\omega t)$.

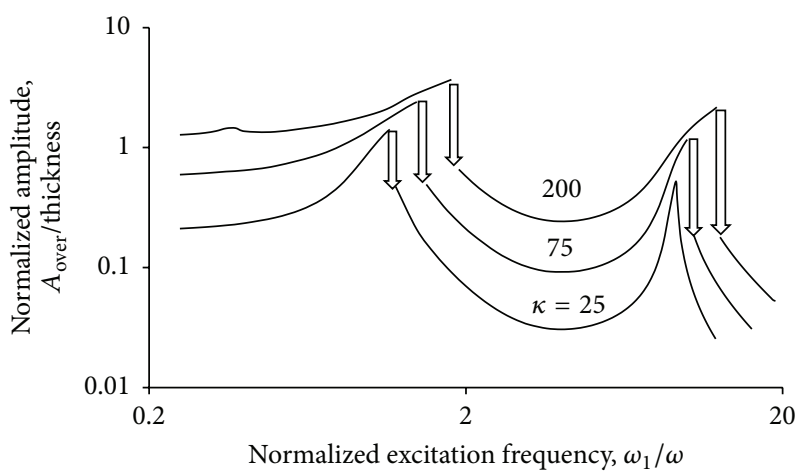

FIGURE 2: Frequency-amplitude curve for various excitation levels.

TABLE 2: (a) Modal contributions for various excitation frequencies, $\omega=1.5 \omega_{1}$. (b) Modal contributions for various excitation frequencies, $\kappa=7.5$.

(a)

\begin{tabular}{lcccc}
\hline $\begin{array}{l}\text { Normalized modal } \\
\text { contribution }\end{array}$ & $\kappa=1$ & 7.5 & 15 & 25 \\
\hline 1st mode & 99.94 & 99.88 & 99.67 & 99.54 \\
2nd mode & 0.05 & 0.10 & 0.29 & 0.40 \\
3rd mode & 0.01 & 0.01 & 0.04 & 0.06 \\
\hline
\end{tabular}

(b)

\begin{tabular}{lcccc}
\hline $\begin{array}{l}\text { Normalized modal } \\
\text { contribution }\end{array}$ & $\omega=0.5 \omega_{1}$ & $\omega_{1}$ & $3 \omega_{1}$ & $6 \omega_{1}$ \\
\hline 1st mode & 99.59 & 99.78 & 96.03 & 78.20 \\
2nd mode & 0.36 & 0.19 & 3.56 & 20.25 \\
3rd mode & 0.05 & 0.03 & 0.42 & 1.55 \\
\hline
\end{tabular}

level and 2nd level solutions are bigger when the excitation level or excitation frequency is higher. It is implied that the higher harmonic components in the vibration responses are more important when the excitation level or excitation frequency is higher. Tables 2(a)-2(b) show the contributions of the first three symmetric modes for various excitation magnitudes and frequencies. It can be seen that the 2 mode solutions are accurate enough for the excitation frequency range from $0.5 \omega_{1}$ to $6 \omega_{1}$ and excitation parameter from $\kappa$ $=1$ to 25 . For the excitation frequency near or less than the 1st resonant frequency, the 1st mode response is dominant. It is implied that the single mode solution is accurate enough. When the excitation frequency is set higher and closer to the 2 nd resonant frequency, the 2 nd mode contribution is more significant. Figures 1(a)-1(b) show the frequencyamplitude curves for two excitation cases (one is uniformly distributed, $F(t)=7.5 \rho_{A} g \sin (\omega t)$; the other is $F(x, t)=$ $\left.(0.1 \sin ((\pi / L) x)+0.9 \sin ((3 \pi / L) x)) 75 \rho_{A} g \sin (\omega t)\right)$. It can be seen that the modified harmonic balance solutions well agree with those obtained from the numerical integration method used in [11]. The well-known jump phenomenon can be seen at the 1st peak. There are only small deviations observations around $\omega=0.5 \omega_{1}$ and $2 \omega_{1}$. In Figure $1(\mathrm{a})$, the 2 nd resonant peak looks like more linear (no jump phenomenon) because the 2 nd modal force is quite small. Thus, it can be considered as linear vibration. In Figure 1(b), the 2nd modal force is set higher to induce the jump phenomenon. Figure 2 shows the overall vibration amplitude plotted against the excitation frequency for various excitation levels. The excitation function is $F(x, t)=(0.1 \sin ((\pi / L) x)+0.9 \sin ((3 \pi / L) x)) \kappa \rho_{A} g \sin (\omega t)$. The jump phenomenon is seen at each peak of the three excitation cases, except the 2 nd peak of $\kappa=25$. Besides, there is a very small damped nonlinear peak observed around $\omega=$ $0.4 \omega_{1}$ due to the high excitation level or high nonlinearity. Figures 3(a)-3(c) show the 1st level residues remained at the 1st and 2nd modal equations for various excitation frequencies. It can be seen that the residues are monotonically increasing with the excitation parameter; the residues in the 1st modal equation are bigger than those in the 2 nd modal equation, and the residues of $\omega=\omega_{1}$ are the highest among the three cases while the residues of $\omega=6 \omega_{1}$ (the excitation 


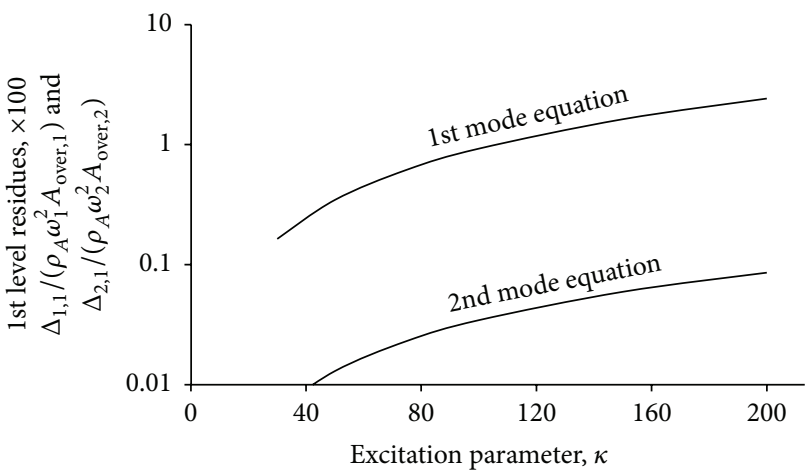

(a)

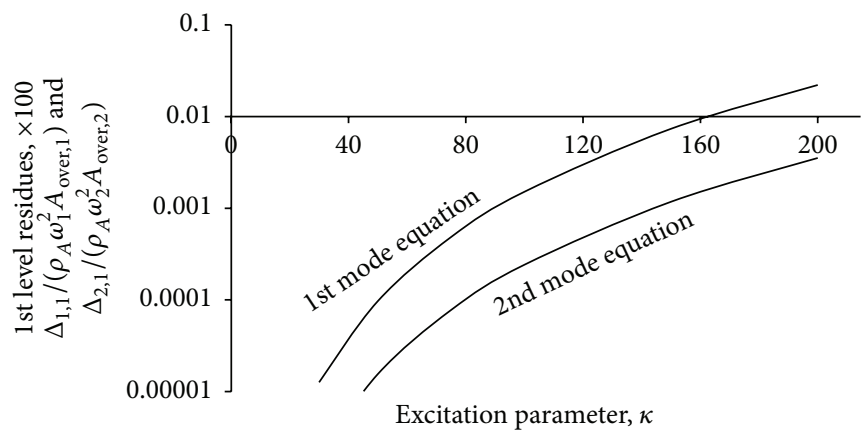

(b)

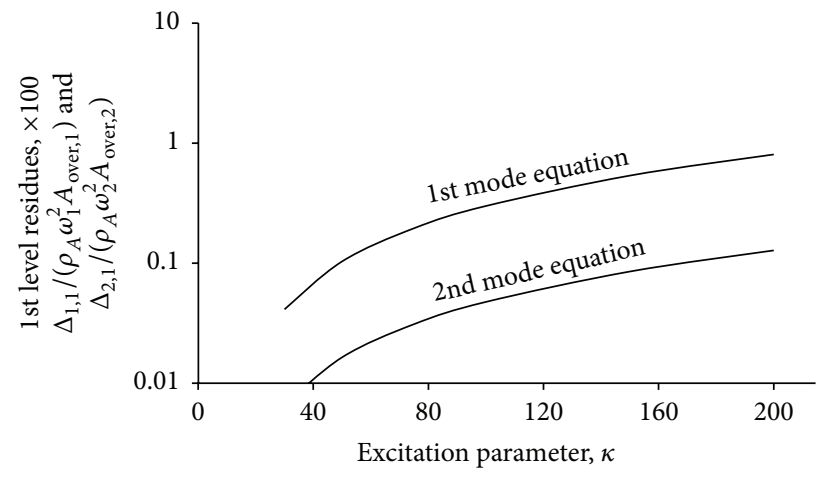

(c)

Figure 3: (a) The 1st level residues remained at the 1st and 2nd modal equations, $\omega=\omega_{1}$. (b) The 1st level residues remained at the 1st and 2nd modal equations, $\omega=6 \omega_{1}$. (c) The 1st level residues remained at the 1st and 2nd modal equations, $\omega=9 \omega_{1}$.

frequency is in between the two resonant frequencies) are the smallest. In general, the results have shown the converged solutions and proven the accuracy of the proposed method.

\section{Conclusions}

In this study, the analytic solution steps for nonlinear multimode beam vibration using a modified harmonic balance approach and Vieta's substitution have been developed. Using the proposed method, the nonlinear multimode beam vibration results can be generated without the need of nonlinear equation solver. The standard simply supported beam case has been considered in the simulation. The solution convergences and modal contributions have been checked. The theoretical result obtained from the proposed method shows reasonable agreement with that from the previous numerical integration method.

\section{Conflict of Interests}

The author declares that there is no conflict of interests regarding the publication of this paper.

\section{Acknowledgment}

The work described in this paper was fully supported by a grant from City University of Hong Kong (Project no. 7004362/ACE).

\section{References}

[1] R. A. Roberts and S. M. Eastbourn, "Modeling techniques for a computational efficient dynamic turbofan engine model," International Journal of Aerospace Engineering, vol. 2014, Article ID 283479, 11 pages, 2014.

[2] L. Maxit, C. Yang, L. Cheng, and J.-L. Guyader, "Modeling of micro-perforated panels in a complex vibro-acoustic environment using patch transfer function approach," Journal of the Acoustical Society of America, vol. 131, no. 3, part 1, pp. 21182130, 2012.

[3] M. Luczak, S. Manzato, B. Peeters, K. Branner, P. Berring, and M. Kahsin, "Updating finite element model of a wind turbine blade section using experimental modal analysis results," Shock and Vibration, vol. 2014, Article ID 684786, 12 pages, 2014.

[4] G. De Gaetano, D. Mundo, F. I. Cosco, C. Maletta, and S. Donders, "Concept modelling of vehicle joints and beam-like structures through dynamic FE-based methods," Shock and Vibration, vol. 2014, Article ID 303567, 9 pages, 2014.

[5] I. V. Andrianov, V. I. Olevs'Kyy, and J. Awrejcewicz, "Analytical perturbation method for calculation of shells based on 2D Pade 
approximants," International Journal of Structural Stability and Dynamics, vol. 13, no. 7, Article ID 1340003, 2013.

[6] A. Marathe and A. Chatterjee, "Wave attenuation in nonlinear periodic structures using harmonic balance and multiple scales," Journal of Sound and Vibration, vol. 289, no. 4-5, pp. 871888, 2006.

[7] B.-Y. Moon and B.-S. Kang, "Vibration analysis of harmonically excited non-linear system using the method of multiple scales," Journal of Sound and Vibration, vol. 263, no. 1, pp. 1-20, 2003.

[8] Y. Y. Lee, "Structural-acoustic coupling effect on the nonlinear natural frequency of a rectangular box with one flexible plate," Applied Acoustics, vol. 63, no. 11, pp. 1157-1175, 2002.

[9] J. L. Huang, R. K. L. Su, Y. Y. Lee, and S. H. Chen, "Nonlinear vibration of a curved beam under uniform base harmonic excitation with quadratic and cubic nonlinearities," Journal of Sound and Vibration, vol. 330, no. 21, pp. 5151-5164, 2011.

[10] A. Y. T. Leung, H. X. Yang, and Z. J. Guo, "The residue harmonic balance for fractional order van der Pol like oscillators," Journal of Sound and Vibration, vol. 331, no. 5, pp. 1115-1126, 2012.

[11] Y. Y. Lee, R. K. L. Su, C. F. Ng, and C. K. Hui, "The effect of modal energy transfer on the sound radiation and vibration of a curved panel: theory and experiment," Journal of Sound and Vibration, vol. 324, no. 3-5, pp. 1003-1015, 2009.

[12] C. Y. Lee and C. Y. Chen, "Experimental application of a vibration absorber in structural vibration reduction using tunable fluid mass driven by micropump," Journal of Sound and Vibration, vol. 348, pp. 31-40, 2015.

[13] D. D. Zhang and L. Zheng, "Active vibration control of plate partly treated with ACLD using hybrid control," International Journal of Aerospace Engineering, vol. 2014, Article ID 432970, 12 pages, 2014.

[14] S. W. Yuen and S. L. Lau, "Effects of inplane load on nonlinear panel flutter by incremental harmonic balance method," AIAA Journal, vol. 29, no. 9, pp. 1472-1479, 1991.

[15] F. Alijani, M. Amabili, and F. Bakhtiari-Nejad, "On the accuracy of the multiple scales method for non-linear vibrations of doubly curved shallow shells," International Journal of NonLinear Mechanics, vol. 46, no. 1, pp. 170-179, 2011.

[16] A. Barari, H. D. Kaliji, M. Ghadim, and G. Domairry, "Nonlinear vibration of Euler-Bernoulli beams," Latin American Journal of Solids and Structures, vol. 8, no. 2, pp. 139-148, 2011.

[17] A. Y. T. Leung and Z. J. Guo, "Feed forward residue harmonic balance method for a quadratic nonlinear oscillator," International Journal of Bifurcation and Chaos, vol. 21, no. 6, pp. 17831794, 2011.

[18] E. W. Weisstein, "Vieta’s Substitution," From MathWorld-A Wolfram Web Resource, http://mathworld.wolfram.com/VietasSubstitution.html. 


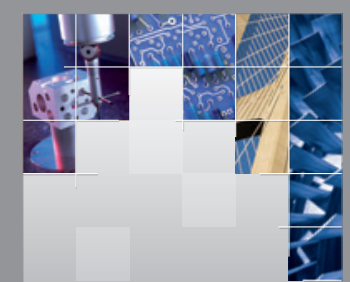

\section{Enfincering}
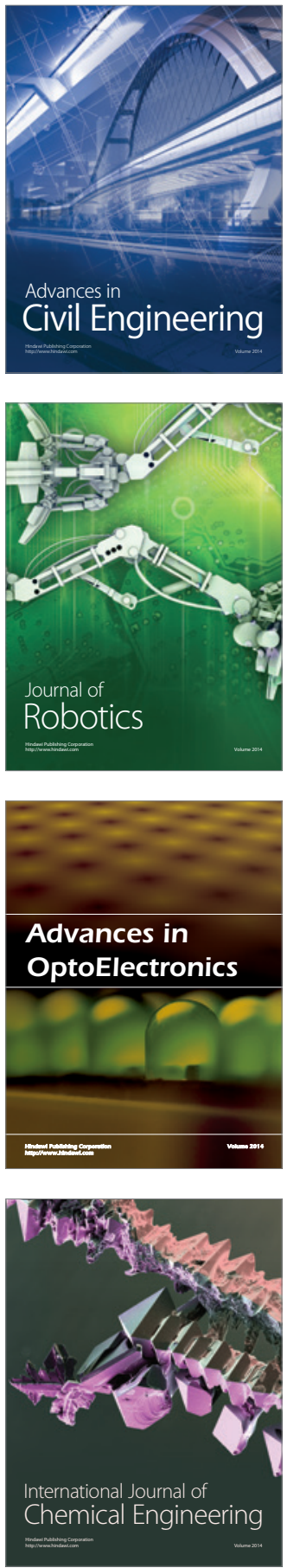

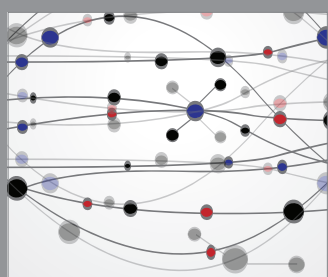

The Scientific World Journal

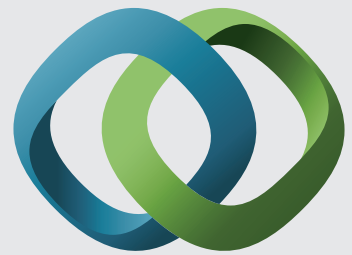

\section{Hindawi}

Submit your manuscripts at

http://www.hindawi.com
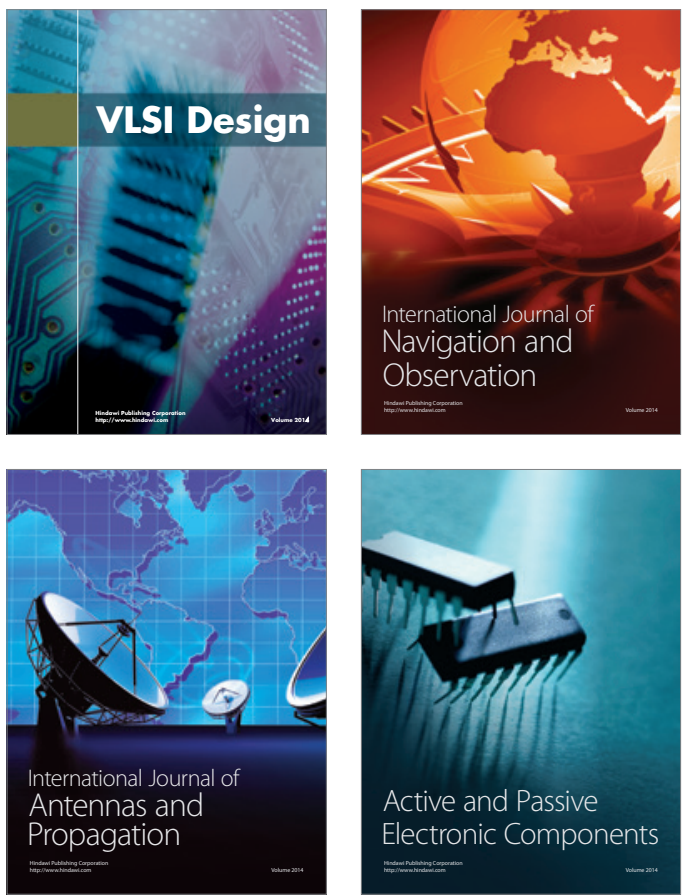
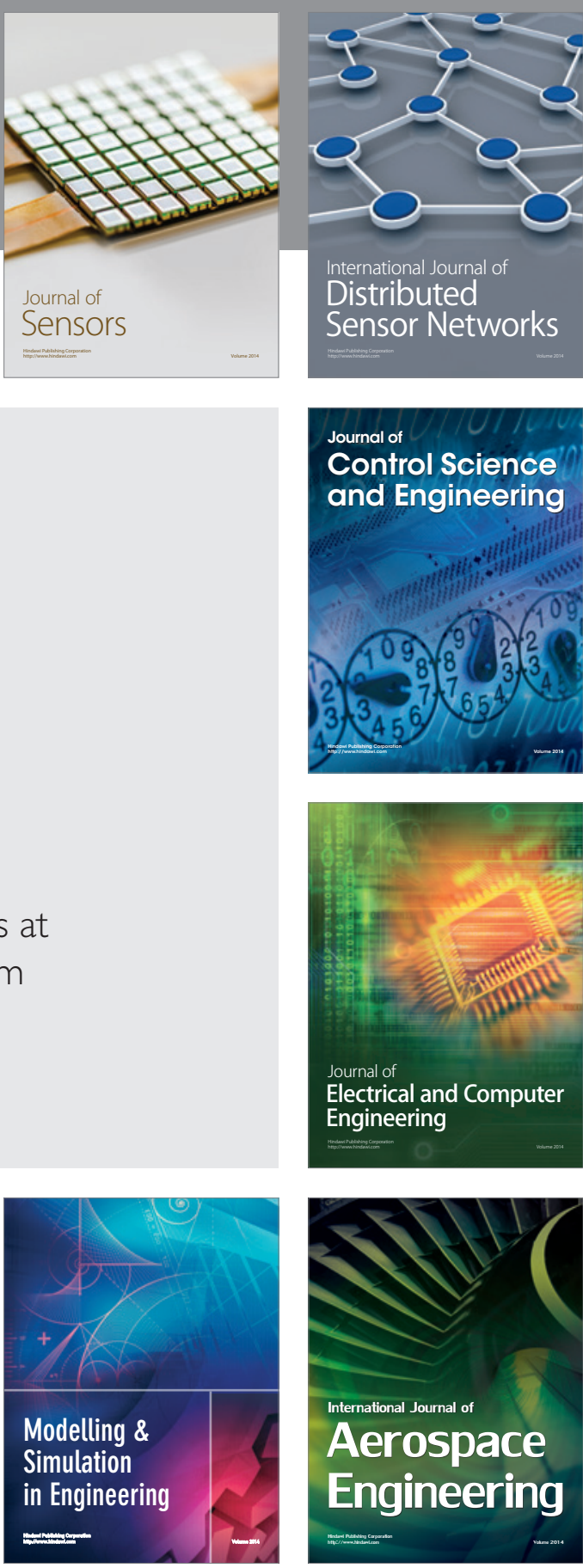

International Journal of

Distributed

Sensor Networks

Journal of

Control Science

and Engineering
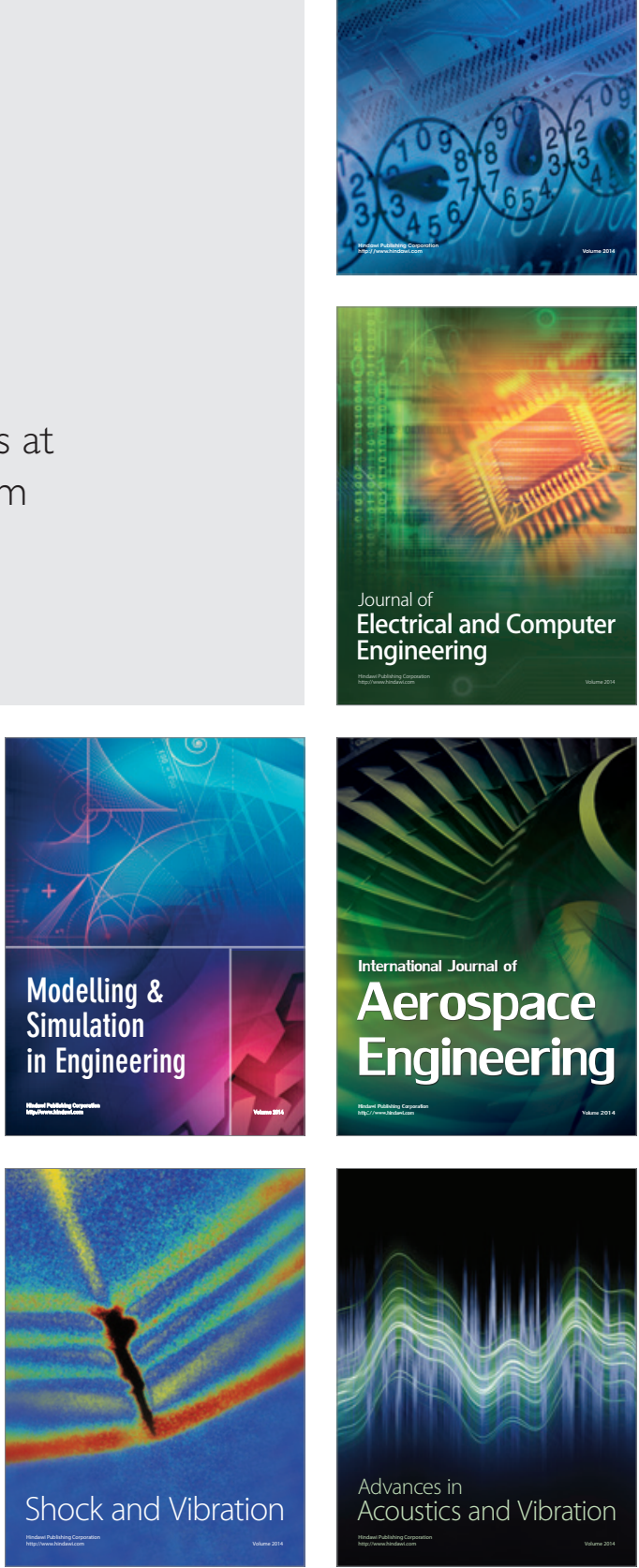